\title{
Erratum to: Evaluation of Vitamin and Trace Element Requirements after Sleeve Gastrectomy at Long Term
}

\author{
Silvia Pellitero ${ }^{1}$ Eva Martínez ${ }^{1} \cdot$ Rocío Puig $^{1} \cdot$ Alba Leis $^{2} \cdot$ Roxanna Zavala $^{3}$. \\ María Luisa Granada ${ }^{2} \cdot$ Cruz Pastor $^{2} \cdot$ Pau Moreno $^{4} \cdot$ Jordi Tarascó $^{4} \cdot$ Jose Balibrea $^{5}$. \\ Manel Puig-Domingo ${ }^{1}$
}

Published online: 22 March 2017

(C) Springer Science+Business Media New York 2017

Erratum to: OBES SURG

DOI 10.1007/s11695-017-2557-1

The name of co-author Jose Balibrea was inadvertently omitted from the original article.

The online version of the original article can be found at http://dx.doi. org/10.1007/s11695-017-2557-1

\section{Silvia Pellitero}

spellitero.germanstrias@gencat.cat

1 Endocrinology and Nutrition Service, Department of Medicine,

Universitat Autònoma de Barcelona, Germans Trias i Pujol,

University Hospital and Research Institute, Carretera de Canyet sn, 08916 Badalona, Spain

2 Clinical Biochemistry Service, Germans Trias i Pujol University, Hospital and Research Institute, Badalona, Barcelona, Spain

3 Endocrinology and Nutrition Service, Hospital Santa Tecla, Tarragona, Spain

4 Surgery Department, Germans Trias i Pujol University, Hospital and Research Institute, Badalona, Spain

5 Endocrine, Metabolic and Bariatric Surgery Unit (EAC-BS Center of Excellence), Vall d'Hebron University Hospital,

08035 Barcelona, Spain 